\title{
O Uso de Ferramentas Estratégicas como Suporte à Gestão do Conhecimento na Educação.
}

\author{
Denize Demarche Minatti Ferreira (UFSC,dminatti@terra.com.br) \\ Jacqueline Keller (UFSC, jacquiekeller@yahoo.com.br) \\ Jacqueline Stumm (UFSC, stumm@sc.senac.br) \\ Neri dos Santos (UFSC, neri@egc.ufsc.br)
}

Resumo: A gestão do conhecimento é um recurso estratégico para a sociedade e tem sua essência pautada no conhecimento e na disseminação deste. Dessa forma, a transferência de conhecimento é de fundamental importância para a evolução dos indivíduos, das organizações e conseqüentemente da sociedade. Dentro desta perspectiva, a produção, preservação e disseminação do conhecimento é um desafio que também deve ser encarado no ambiente educacional, por este ser reconhecido como local de produção e transferência de conhecimento tornando-se necessário fornecer meios de acesso a estes dados à comunidade acadêmico-científica. Tais ações estão ligadas, dentre outras formas, ao uso das Tecnologias da Informação (TI) que podem fornecer suporte a gestão do conhecimento no âmbito da educação. Nesse sentido, este artigo objetiva a descrição de três ferramentas que podem ser utilizadas para gerir conhecimento no âmbito da educação. Como resultado de uma pesquisa bibliográfica, o artigo ressalta a necessidade das escolhas das ferramentas de TI e tem como foco o contexto operacional da instituição, as pessoas envolvidas no processo e principalmente o objetivo da instituição de ensino com o seu uso.

Palavras-chave: tecnologia da informação, ferramentas estratégicas, gestão do conhecimento, educação.

Abstract: The knowledge management is a strategical resource for society and has its essence based on knowledge and this dissemination. Thus, the knowledge transfer is of fundamental importance to the development, organizations and, therefore society. Inside this perspective, the knowledge production, preservation and dissemination are challenge that also be seen in the educational environment for this be recognized as local of production and knowledge transfer becoming necessary to supply access to the academic community - scientific data. Such actions are linked, in other ways, the use of Information Technology providing support for Knowledge Management in education scope. The article aims three tools descriptions that can be used to manage knowledge in education. As result, the article stands out the necessity choices of Information Technology tools to have as focus the institutional operational context, the people involved and mainly objective of education institution with its use.

Keywords: information technology, strategies tools, knowledge management, education.

\section{INTRODUÇÃO}

O desenvolvimento das sociedades pautou-se na comunicação e na transferência de conhecimento, as quais aconteceram de diversas formas e foram evoluindo com o tempo. O conhecimento sempre foi importante, é um recurso proeminente, sendo muitas vezes considerado mais importante até mesmo do que a matéria prima. Dessa forma, pode-se referir a informação e conhecimento como 
produtos econômicos e entender porque é prioritário gerenciar esse ativo de forma correta.

A geração do conhecimento é feita pelo ser humano, acumulado e administrado pela sociedade e por ele operacionalizado para satisfação de suas necessidades. As organizações, que são sociedades de pessoas com objetivos bem definidos, reúnem e operacionalizam especialidades de conhecimento para, deste modo, alcançar maior eficiência e eficácia na gestão desses com o intuito de atingir seus objetivos e cumprir sua missão (SANTIAGO E SANTIAGO JR, 2007).

Mudanças profundas em competitividade fizeram com que a maioria das organizações entendesse conhecimento com um recurso estratégico e que este lhes oferece uma vantagem competitiva. Nesta visão de conhecimento como vantagem competitiva, é necessário gerenciar conhecimento de forma sistemática e eficiente. Stewart (1998) diz que as mudanças que nos cercam não são meras tendências, mas fruto de forças poderosas e incontroláveis: a globalização, que abriu enormes mercados, mas também como corolário necessário gerou uma gigantesca quantidade de concorrentes; a disseminação da Tecnologia da Informação (TI) e o crescimento das redes de computadores, fundamentais para o compartilhamento do conhecimento. A esse respeito, o autor ainda informa o surgimento da economia da nova Era da Informação, tem como fontes fundamentais; o conhecimento e a comunicação, e não recursos naturais ou o trabalho físico.

Atualmente não há uma organização que não faça uso intensivo da informação e que não tenha se tornado dependente do conhecimento e das ferramentas de TI para sua criação e seu gerenciamento. Como em outros segmentos, a educação está sujeita as mesmas pressões do mercado, o que reforça a necessidade das instituições de ensino pensarem como negócio e adotarem técnicas para criar e gerir seu conhecimento. Percebida a necessidade pergunta-se, como a TI pode fornecer suporte à gestão do conhecimento no âmbito da educação? Quais são as ferramentas adequadas às necessidades das instituições de ensino e que devem ser adotadas para gerir seu conhecimento?

Busca-se, neste artigo, resultado de uma pesquisa bibliográfica, apresentar três ferramentas de suporte a gestão do conhecimento, além de destacar qual o foco de gestão de cada uma delas, ressaltando que a escolha das ferramentas de TI devem ocorrer de acordo com a estratégia adotada e necessária à particularidade de cada instituição de ensino.

$\mathrm{Na}$ literatura pertinente, encontram-se diferentes ferramentas de gestão do conhecimento, mas para este artigo priorizou-se a descrição e o estudo da aplicabilidade de Repositórios Institucionais (RI), Árvores do Conhecimento e dos Ambientes Colaborativos como ferramentas estratégicas à gestão do conhecimento no âmbito da educação.

\section{MATERIAIS E MÉTODOS}

Para o cenário apresentado, o artigo faz uma revisão bibliográfica e foi divido em quatro etapas. Inicialmente descreve a gestão do conhecimento e na segunda etapa, sua importância no âmbito educacional. Na seqüência apresenta as três ferramentas de gestão do conhecimento e seu foco de utilização. E por fim, apresenta-se uma discussão relacionando os contextos abordados. 


\section{SUPORTE TEÓRICO}

\subsection{GESTÃO DO CONHECIMENTO}

As organizações, independentes da sua área de atuação, geram e usam conhecimento. Como as organizações interagem com o seu ambiente, elas absorvem informação, as transformam em conhecimento e seus tomadores de decisão executam ações baseadas numa combinação das suas experiências, valores, regras internas, profundamente influenciadas pela sua cultura organizacional (JOÃO, 2005). Diz que sem conhecimento, resultante da prática e da aprendizagem, uma organização não poderia se organizar, e estaria impossibilitada de se manter como uma organização sustentável e criadora de conhecimento.

Para Corso et al (2006) gestão do conhecimento refere-se a criação de um ambiente que encoraje as pessoas a aprender e compartilhar conhecimento através do alinhamento de objetivos, integrando unidades de informação dentro e fora dos limites da organização e produzindo novos conhecimentos que sejam úteis e passíveis de uso pela mesma. Já Schultz e Leidner (2002) dizem que a gestão do conhecimento, resumidamente, pode ser entendida como a geração, representação, armazenamento, transferência, transformação, aplicação, adaptação e proteção do conhecimento organizacional.

Nonaka e Takeuchi (1997), Sveiby (1998), Davenport e Prusak (1998), Kanter (1999), Eboli (1999), Cavalcanti, Gomes e Pereira (2001) e Yang (2004), citados por Leitão (2006) colocam que a gestão do conhecimento traz uma nova luz sobre como uma organização pode ganhar vantagem competitiva e tornar-se inovativa com sucesso. E isso, na opinião de Del Tio (2006), é transformado no que hoje chama-se de "gestão do conhecimento", a qual contribui para o envolvimento de todas as pessoas dentro de uma organização, empresas ou instituição, unificando as participações ativas e de opiniões, potencializando os resultados obtidos e a eficácia da organização.

Para Nonaka e Takeuchi (1997) a criação do conhecimento organizacional baseia-se num "framework" com duas dimensões: a epistemológica que mostra que apenas indivíduos criam conhecimento, e a ontológica que se refere a interação entre conhecimento tácito e explícito. Essas duas dimensões servem de base para a definição dos quatro processos de criação de conhecimento:

1. A socialização que se refere ao compartilhamento de idéias.

2. A externalização que é o processo de criação do conhecimento (articulação de conhecimento tácito em conceitos explícitos).

3. A combinação que sistematiza conceitos em um sistema de conhecimentos e;

4. A internalização que converte conhecimento explícito em tácito.

Santiago e Santiago Jr. (2007) afirmam ser importante para qualquer organização que os conhecimentos aprendidos e/ou adquiridos estejam ao alcance de todos. As informações devem estar à disposição, serem acessíveis a todo o momento, para qualquer colaborador e em qualquer etapa de atividade dos processos existentes. Os conhecimentos que estão exclusivamente nas cabeças das pessoas necessitam de processos mais complexos para sua "captura", também devem ser geridos e resgatados, por meio de contatos diretos e muitas vezes informais, possibilitando comunicação, troca de informações e experiências.

Nonaka e Takeuchi (1997), Probst et al. (2002) e Heisig (2001) vêem a gestão do conhecimento como um ciclo dinâmico e em constante evolução. Desta forma, criar e implementar processos de criação, armazenamento, gerenciamento e disseminação do 
conhecimento passam a representar um importante desafio enfrentado pelas organizações, assim, para uma eficaz gestão do conhecimento é necessário associá-la a gestão de pessoas e a gestão da informação apoiada no uso de tecnologias e ferramentas estratégicas.

\subsection{GESTÃO DO CONHECIMENTO NA EDUCAÇÃO}

Tradicionalmente, a produção de conhecimento científico ocorre principalmente nas universidades, tornando o ambiente educacional um espaço reconhecido como local de produção e transferência de conhecimento.

Assim como em qualquer organização, a gestão do conhecimento deve ser objetivo fundamental de qualquer instituição educacional, como aprender é a sua função central, deve ser refletida em como a organização opera. Deve-se pensar numa estratégia de gestão do conhecimento direcionada com o que a instituição quer alcançar, considerando as pessoas envolvidas, o contexto operacional da organização a história e a capacidade.

Gestão do conhecimento também é usada para encorajar organizações à criação e compartilhamento de conhecimento resultando em melhorias em produtividade, inovação, competitividade e melhores relacionamentos entre as pessoas nessas organizações.

No entanto, alguns problemas são enfrentados para tornar a gestão do conhecimento eficiente no âmbito educacional:

1. Melhorar o acesso à pesquisa e a dados de modo geral.

2. Suprir a demanda de mecanismos que garantam a realização efetiva do todos os processos relacionados à produção até o uso do conhecimento e;

3. Ainda possibilitar a coletivização e o compartilhamento do conhecimento.

Para resolver tais problemas depara-se com vários desafios: a necessidade de implementar, aprimorar e potencializar a transferência de conhecimento o que conseqüentemente maximizaria a criação de novos conhecimentos otimizaria recursos, promoveria o crescimento das instituições educacionais e traria o avanço da ciência.

Dentro desta perspectiva, existe a necessidade da busca de novas estratégias que ofereçam suporte à gestão do conhecimento. Ubon e Kimble (2002) sugerem que uma iniciativa eficiente de gestão do conhecimento requer estratégias, dentro delas o uso de ferramentas para gestão.

\subsection{FERRAMENTAS ESTRATÉGICAS PARA GESTÃO DO CONHECIMENTO}

Sob o ponto de vista de Ubon e Kimble (2002) uma iniciativa eficiente de gestão do conhecimento requer a aplicação de um grande conjunto de ferramentas e técnicas de gestão. Segundo os autores, o uso da TI para a gestão do conhecimento pode ser definido pelas ferramentas utilizadas para gerenciar o conhecimento explícito na organização permitindo, dessa forma, a ampliação do alcance e aceleração da velocidade de transferência desse conhecimento.

\subsubsection{Repositórios Institucionais}

É freqüente nas instituições de ensino superior a necessidade de gerenciar a atividade educacional e a produção científica de forma eficaz e transparente. Ao permitir que seus dados fiquem disponíveis em repositórios, estas instituições apóiam o 
desenvolvimento de novas relações entre a comunidade acadêmica e centros de pesquisa nacionais e internacionais, ação esta que tem como resultado o estímulo econômico e o desenvolvimento social e pedagógico.

Os primeiros repositórios surgiram da necessidade do homem armazenar informações, soma-se a este fato, a posterior, a crescente necessidade de se obter informações referenciais, de caráter científico, advindas de confiáveis instituições de ensino. A partir do ano 2000, a facilidade em criar e disseminar conteúdos sob a forma de informações digitais aumentou rapidamente, desafiando as universidades e institutos de pesquisa e de publicação científica a coordenar, compartilhar e preservar seus dados em formato digital.

Segundo Gonzaléz e Porcel (2007) um Repositório Institucional é um arquivo eletrônico de toda a produção científica de uma instituição, armazenada num formato digital, que permite a busca e a recuperação para seu posterior uso tanto no âmbito nacional quanto internacional.

Em 2002, foi lançado o primeiro Repositório Digital, o DSpace, um projeto desenvolvido pelo Instituto de Tecnologia de Massachusetts (MIT), em colaboração com a Hewlett-Packard, para preservar, recolher, gerenciar e permitir conhecer a produção intelectual da instituição. O DSPace, que é disponibilizado livremente às instituições, pode ser adaptado e expandido e é uma plataforma de software que possibilita (MCLENDON, 2005):

a) Capturar e descrever documentos digitais de acordo com um workflow adaptável aos processos específicos de uma comunidade.

b) Distribuir os documentos digitais da instituição na Web, possibilitando a pesquisa e obtenção de cópias aos utilizadores.

c) Preservar os documentos digitais em longo prazo.

Para Mclendon (2005) a criação de RI's multidisciplinares para bibliotecas, arquivos e centros de pesquisa representou uma nova estratégia para o gerenciamento do conhecimento produzido pelas universidades.

Segundo Lynch (2003), os RI's desenvolvidos pelas universidades são considerados como "um conjunto de serviços que a universidade oferece aos membros de sua comunidade, visando o gerenciamento e a disseminação dos materiais (informações) digitais criados pela instituição e pelos membros de sua comunidade".

Rodrigues (2007) os define como sistemas de informação que armazenam, preservam, divulgam e dão acesso à produção intelectual de comunidades universitárias e/ou de um grupo específico de instituições ou pessoas. Cita também que estes repositórios contribuem para o aumento da visibilidade e do valor público das instituições, servindo como indicador tangível da sua qualidade e contribuindo assim para a reforma do sistema de comunicação científica, expandindo o acesso aos resultados da pesquisa e reassumindo o controle acadêmico sobre a publicação científica.

Corroborando com os autores citados, Costa e Leite (2006) consideram os RI's como sistemas de informação que servem para armazenar, preservar e difundir os resultados de pesquisa e desenvolvimento de uma determinada instituição e/ou de um determinado grupo de instituições, mantidos de forma individualizada ou por grupos de instituições que trabalhem de forma cooperativa.

Os autores afirmam ainda que os RI's apresentam duas funções fundamentais: a primeira, na melhoria do ensino, do aprendizado e da pesquisa, ou seja, na melhoria do saber e da comunicação científica. E em segundo, no potencial que encerram como 
instrumentos de gestão do conhecimento produzido, disseminado e utilizado nas e pelas universidades.

Os RI's constituem uma manifestação evidente da importância emergente da gestão do conhecimento no contexto da educação superior. Com base na similaridade e complementaridade existente entre os processos do sistema de comunicação científica e as atividades da gestão do conhecimento, os repositórios institucionais como uma poderosa alternativa tanto para a comunicação quanto para a gestão do conhecimento científico (COSTA E LEITE, 2006).

Cabe citar que para qualquer instituição educacional um RI é um elemento central e importante na estratégia da gestão do conhecimento, pois forma um sistema de gestão de conteúdos, uma vez que além de documentos, oferece também à comunidade acadêmica, serviços para a gestão de sua produção cientifica.

Deste modo, os RI's auxiliam de forma extremamente importante a gestão do conhecimento, não somente em sua própria abordagem, mas também no sentido de preservar a memória organizacional de uma instituição.

\subsection{2 Árvores do Conhecimento}

A única saída para que as organizações possam de fato "saber o que sabem" é por meio da coletivização e do compartilhamento do conhecimento permitindo, assim, que os membros de uma determinada comunidade possam revelar suas qualificações e habilidades e mostrá-las à organização.

Como proposta a esse problema de coletivização e compartilhamento do conhecimento surgiram as Árvores de Conhecimento. Desenvolvidas por Pierre Lévy, e Michel Authier, têm como fundamento o princípio que ninguém sabe tudo e que cada um sabe alguma coisa (LÉVY E AUTHIER, 2000).

A idéia que toda e qualquer organização ou comunidade necessita antes de tudo saber o que ela sabe é um dos princípios da gestão do conhecimento. Isto só é possível se tivermos o mapeamento e exploração do conhecimento existente em seus diferentes âmbitos e, principalmente, nas pessoas que compõem esta organização ou comunidade.

As Árvores de Conhecimento trazem em sua estrutura conceitual e operacional conceitos importantes que vem de encontro aos princípios da gestão do conhecimento. Isto fica mais visível a partir do reconhecimento de um de seus fundamentos que prima pela possibilidade de dar visibilidade e reconhecimento a cada pessoa que está presente no coletivo (MOURA, s.d). Essa visão é buscada em diversos momentos por diferentes organizações por permitir a possibilidade do reencontro da coletividade e de uma sociedade humana e concreta.

Segundo Lévy e Authier (2000), as Árvores de Conhecimentos são uma hipótese de democracia que se encaixa na atual sociedade voltada para a informação e a comunicação rápida.

Instituições de ensino e outros tipos de organizações podem fazer o gerenciamento global de suas competências por meio das Árvores de Conhecimento. Em um espaço que relaciona o conjunto de competências de uma comunidade há a possibilidade de cada membro dessa comunidade reconhecer a diversidade de suas competências, mesmo aquelas que não são validadas por sistemas escolares e universidades clássicas. Podem mapear também as competências de diferentes grupos, o que facilita os indivíduos aprender uns com os outros viabilizando a construção de novas competências permitindo assim a abertura e a coletivização do conhecimento (BARROS, 2000). 
Neste sentido, cada grupo possuirá uma árvore que corresponde à sua realidade sendo que a organização dessa árvore não está fixada, pois a árvore vai se transformando à medida que se transformam as competências da própria comunidade. Ela reflete a experiência coletiva de um grupo e, portanto, vai evoluir com esta experiência (LÈVY, 1997).

Nas comunidades de saber, cada indivíduo é considerado uma singularidade, e a comunidade, por sua vez, é uma composição de singularidades. Os saberes dos indivíduos são definidos por eles mesmos com base em suas experiências e seus conhecimentos.

Portanto, as Árvores de Conhecimento valorizam as riquezas humanas em sua variedade e diversidade, dando condições de reconhecimento dos conhecimentos que estão além dos saberes oficiais, permitindo aprendizagem de saberes que podem ultrapassar àquelas disponíveis pela comunidade.

\subsubsection{Ambientes Colaborativos}

Membros de equipes, independentemente de sua localização geográfica, necessitam se comunicar, compartilhar e transferir conhecimento Os Ambientes Colaborativos, também conhecidos como groupware, apóiam esse trabalho coletivo.

Dessa forma, tais ambientes necessitam do uso apropriado e integrado de um conjunto de ferramentas de TIC's a fim de desenvolver uma rede de contatos interna e externa à organização.

Quando se fala em Ambientes Colaborativos de aprendizagem, faz-se referência àqueles onde seja possível que diferentes usuários participem, colaborem ou cooperem, sempre no sentido de uma produção que represente o objetivo em comum da ação. Importante reforçar que a interação se dá entre as pessoas favorecidas pelos recursos fornecidos pelo uso do computador para viabilizar, intensificar ou mesmo armazenar o conhecimento decorrente dessa interação.

As TI's têm favorecido muito a expansão de comunidades de aprendizagem. Atualmente, muitos ambientes virtuais de aprendizagem são utilizados não só para disponibilizar conteúdos, mas também para promover e gerenciar processo de ensinoaprendizagem. Esses ambientes são conhecidos também por Sistemas de Gerenciamento de Cursos, do inglês Learning Management System (LMS).

Vários ambientes virtuais, sistemas de interação, softwares para gerenciamento de informação e troca têm promovido a rápida expansão de comunidades virtuais de aprendizagem. Muitas comunidades são divulgadas na própria Web e por serem abertas permitem o ingresso de participantes interessados em aprender assuntos em comum. $\mathrm{O}$ livre acesso a conteúdos, recursos, softwares e sistemas de interação e gerenciamento ampliaram a circulação e construção do conhecimento (WILLINSKY, 2006).

As tecnologias colaborativas estão relacionadas ao treinamento baseado na Web, com ambientes virtuais em que se utilizam a telecomunicação por meio da internet e de multimídia. Elas permitem estratégias de discussão em grupos com mediação de tutor à distância e utilização de jogos interativos, ou seja, a interação que ocorre não é apenas do indivíduo com a tecnologia, mas também entre indivíduos. O conhecimento é compartilhado por meio da colaboração dos indivíduos, gerando novos conhecimentos (CASTRO E FERREIRA, 2006).

O conhecimento é a âncora de um ambiente colaborativo e esse ambiente deve proporcionar a representação e a ação de toda atividade presente nas relações entre os indivíduos, sem que isto modifique ou desequilibre este ambiente. É importante também que o trabalho de cada um para construção do produto final possa ser resgatado por 
todos permitindo o acompanhamento e avaliação do processo de construção, levando à valorização do indivíduo enquanto integrante do grupo.

\section{RESULTADOS}

Em relação ao uso de ferramentas de TI no suporte à gestão do conhecimento na educação pode-se observar que:

$\checkmark$ Fortalecem a perspectiva de produção, preservação e disseminação do conhecimento, em um ambiente educacional reconhecido como local de produção e transferência de conhecimento.

$\checkmark$ Com a utilização destas ferramentas, o desafio está em implementar, aprimorar e potencializar a transferência do conhecimento para maximizar a criação de novos conhecimentos e se alcançar o êxito.

Quanto às ferramentas de TI apresentadas:

$\checkmark$ Observou-se que cada uma das ferramentas apresentadas deve ser utilizada em uma estratégia específica, sendo assim, a escolha pelo uso de uma ferramenta em detrimento de outra deve estar focada no contexto operacional da instituição, nas pessoas envolvidas no processo e principalmente no objetivo da instituição de ensino com o seu uso.

$\checkmark$ O uso de Repositórios Institucionais deve ser pensado na medida em que a instituição deseje preservar sua memória.

$\checkmark$ Árvores de Conhecimento são desejáveis quando instituições de ensino desejam coletivizar o conhecimento dos indivíduos que as compõem, possibilitando dar visibilidade e reconhecimento a cada pessoa que está presente no coletivo.

$\checkmark$ Ambientes Colaborativos apóiam o trabalho coletivo possibilitando aos membros das equipes, independentemente de sua localização geográfica, se comunicar, compartilhar e transferir conhecimento.

\section{CONSIDERAÇÕES FINAIS}

O objetivo do artigo, como abordado em sua apresentação, foi o de mostrar como a TI pode fornecer suporte à gestão do conhecimento no âmbito educacional por meio da utilização de algumas de suas ferramentas.

Verificou-se que as ferramentas de TI podem ainda apoiar a autonomia das pessoas, eliminarem barreiras de aprendizagem e neutralizarem fronteiras geográficas da distancia, auxiliar nas decisões e na resolução de problemas; viabilizarem o trabalho em grupo, permitirem o resgate de experiências e a localização de peritos, e muito mais.

A TI deve compreender e considerar que as pessoas são as únicas capazes de produzir o produto e o objeto da gestão do conhecimento, justamente, o conhecimento, a matéria prima mais literal de uma instituição. Deve-se considerar ainda que a gestão do conhecimento requer uma abordagem muito mais humana do que tecnológica. Só depois de entender essa realidade é que uma instituição poderá realmente empregar seus produtos, serviços e soluções em gestão do conhecimento, de alta qualidade, 
responsabilidade e que realmente cumpram seus propósitos. Transformar uma decisão de TI em sucesso na gestão do conhecimento depende muito da cultura organizacional, de visão e alinhamento estratégico, do envolvimento das pessoas, do comprometimento dos usuários, do contexto e significado e do valor agregado ao trabalho e aos negócios.

\section{REFERÊNCIAS BIBLIOGRÁFICAS}

BARROS, M. E. B. de. Procurando outros paradigmas para a educação. Educ. Soc. vol.21 no.72 Campinas Agosto, 2000.

CASTRO, M. N. M.; FERREIRA, L.D.V. TD\&E a distância: múltiplas mídias e clientelas. In: BORGES-ANDRADE, J. E. et al. Treinamento, desenvolvimento e educação em organizações e trabalho: fundamentos para a gestão de pessoas. Porto Alegre: Artmed, 2006.

COSTA, S. M. de S.; LEITE, F. C. L. Repositórios institucionais: potencial para maximizar o acesso e o impacto da pesquisa em universidades. In: Conferência Iberoamericana de Publicações Eletrônicas no Contexto da Comunicação Científica, 1. Brasília, Universidade de Brasília, 25 a 28 de abril de 2006.

CORSO, M., et. al. Managing dispersed workers: the new challenge in Knowledge Management. Technovation, v.26, p.583-594, 2006.

DEL TIO, A. L. Gestão do conhecimento aplicado ao planejamento estratégico em instituições de ensino superior. Campinas, 2006. Dissertação de mestrado. PUC Campinas, 2006.

GONZALÉZ, A. B.; PORCEL, A. F. Diretrizes para Criação de Repositórios Institucionais nas Universidades e Organizações de Educação Superior. Disponível em: <http://infolac.ucol.mx/observatorio/Directrizes_RI_portugues.pdf.>Acesso em: 10 jun. 2008.

HEISIG, P., MERTINS, K. E VORBECK J. Knowledge Management. Concepts and Best practices in Europe. Second edition. Springer-Verlog. Berlin Heidelberg. New York, 2001.

JOÃO, B. M. Estratexias de valor para a creación de coñecemento en organizacións intensivas en coñecemento. Revista Galega de Economía, vol. 14, núm. 1-2 (2005), pp. $1-19$.

LEITÃO, J. S. de Sá. Estratégias para facilitar o compartilhamento de conhecimentos em uma organização de pesquisa e desenvolvimento. 2006. Tese (Doutorado) - Programa de Pós-Graduação em Engenharia de Produção. Universidade Federal de Santa Catarina, Florianópolis, 2006.

LÉVY, P.; AUTHIER, M. As árvores de conhecimentos. Pref. de Michel Serres. 2a. ed. São Paulo, Escuta, 2000.

LÈVY, P. Árvores de Conhecimento para inteligência coletiva na educação e na formação. Texto extraído de Lèvy, P. Ciberculture, Paris. Ed. Odile Jacob, Ed. Du Conseil de Lénvelope, 1997, cap. XII, pp. 214-222. Disponível em: $<$ http://www.ddic.com.br/arquivos3.php>. Acesso em: 10 jun. 2008.

LYNCH, C. A. Institutional Repositories: essential infrastructure for scholarship in the digital age. ARL [Association Research Libraries], n. 226, feb. 2003, p 1-7. Disponível em: <http://www.arl.org/resources/pubs/br/br226/br226ir.shtml>. Acesso em: 10 jun. 2008.

MCLENDON, W. Institucional Repositories. Scholarly Communications in a Digital World. jan. 2005. Disponível em: <http://www.unc.edu/scholcomdig/whitepapers/mclendon.pdf > Acesso em: 10 jun. 2008 
MOURA, A. H. de. As árvores de conhecimento e a gestão estratégica de informações do conhecimento. Disponível em: $<$ http://www.ddic.com.br/arquivos1.php> Acesso em 10 jun. 2008.

NONAKA, I.; TAKEUCHI, H. Criação de conhecimento na empresa. Rio de Janeiro: Elsevier, 1997 - $18^{\mathrm{a}}$ Reimpressão.

PROBST, G. Managing Knowledge, Building Blocks for Success. Wiley. West Sussex, England, 2002.

RODRIGUES, E. Promovendo o acesso livre à literatura científica: o RepositóriUM e a Política de Auto-Arquivo da Universidade do Minho. In: Encontro sobre Repositórios Institucionais, Instituto Politécnico de Leiria. Leiria, Portugual, 09/05/2007.

SANTIAGO JR, J. R. S.; SANTIAGO, J. R. S. Capital intelectual: o grande desafio das organizações. São Paulo: Novatec Editora, 2007.

SCHULTZ, U.; LEIDNER, D. E. Studying knowledge management in information systems research: Discourses and theoretical assumptions. MIS Quarterly, v.26, n.3, p.213-242, Setembro, 2002.

STEWART, T. A. Capital intelectual: A nova vantagem competitiva. Rio de Janeiro: Elsevier, 1998. 14ª Reimpressão.

UBON, A. Na; KIMBLE, C. Knowledge Management in Online Distance Education. Networked Learning, 2002

WILLINSKY, J. The Access principle: the case for open Access to research and scholarship. Cambridge: MIT, 2006. 